\title{
Response of structure models to sinusoidal dynamic action
}

\author{
E.E. Yurchenko ${ }^{1, \square}$, E.A. Yurchenko ${ }^{1}, A . A$. Kakosyan $^{1}$, L.U. Ovchinnikova ${ }^{1}$, \\ V.E.Yurchenko ${ }^{1}$, and E.K. Birukbaev ${ }^{1}$ \\ ${ }^{1}$ Sochi State University, 94 Plastunskaya st., 94, Sochi, 354000, Russia
}

\begin{abstract}
The use of small-sized models of structures printed on 3-D printers is possible due to the electroelastic properties of ABS plastic. A comparison of their reactions with analogs made of plexiglas, used in studies of concrete structures, was made. The influence of the environment - water or sea sand, support conditions - free support or pinching, shape - simple or complex in plan- on the response of models from two different materials was investigated. The laboratory setup simulated a dynamic sinusoidal effect using: a digital frequency generator from 1 to $10000 \mathrm{~Hz}$, an acoustic system diffuser, a microphone amplifier, two electrodes, and a computer in a twochannel oscilloscope mode. The vibration characteristics at the base and top of the models were recorded. The experimental system turned out to be sensitive to the shape, methods of fastening elements, the structure as a whole, and the medium of wave propagation. The response was analyzed in terms of the dynamic compliance coefficient and resonance frequencies, reflecting the similarity of the responses of the plexiglas and ABS models. Printing will allow you to adjust the shape and connections of the elements of the models so that they work like mechanical resonators - filters - in a narrow band. No resonances are expected outside this band. In the operated structures, the quality of the element connections is reproduced. Changing the existing rigidity to obtain resonances in a narrow band can serve as a criterion for choosing an effective amplification method.
\end{abstract}

\section{Introduction}

According to [1], under conditions of dynamic impacts of the sea or earthquakes, any structure is permissible to represent it as a chain of acoustically coupled mechanical resonators. Wave processes in such systems are characterized by spatial and temporal periodicity, wavelength and frequency, propagation velocity, amplitude and vibration energy. The sensitivity of measuring devices that implement wave processes should take into account the features of wave propagation in various media, materials and the shape of the resonator models. Perturbations proportional to the applied force are considered elastic mechanical waves. Oscillations of particles of an elastic medium, propagating from a generator of electrical oscillations in the form of waves in air, water, sand and models in experiments to an audible sound with a frequency of oscillations from $16 \mathrm{~Hz}$ to $20 \mathrm{kHz}$. The electro elastic

${ }^{*}$ Corresponding author: wsonormalno@yandex.ru 
properties of plexiglass [2] and ABS [3] materials are used to measure the reactions of models - linear dimensions during forced vibrations [4] based on a change in an alternating electric field. The sensitivity of the obtained frequency response allows the use of small-scale modeling of structure prototypes for the choice of shape and structure during design. When assessing defects of elements, soil conditions, joints in the nodes of operated structures, the quality criterion is the dynamic coefficient of compliance used in mechanics [5].

\section{Materials and methods}

For implementation the goal of the research - the response of structures depending on the shape, quality, and soil conditions - a laboratory setup was assembled. It provides the measurement and construction of the frequency response of small-sized models from electro elastic materials based on additive technologies, as in [6]. Prototypes of coastal hydraulic structures are modeled. The installation consists of a frequency generator, an electroacoustic transducer, a box with sand or distilled water, a microphone amplifier with electrodes, a model, and an oscilloscope computer.

1. Frequency generator - creates mechanical sound vibrations - sinusoidal waves. With a smooth mode of switching frequencies from 1 to $10000 \mathrm{~Hz}$, the sensitivity of the models' response to the support conditions was determined. In the mode of switching frequencies from $1 \mathrm{~Hz}$ to $10 \mathrm{kHz}$ with an interval between $\mathrm{kHz}$ of $1-2 \mathrm{~s}$ (Figure 1), the frequency response of the models was obtained.

Table 1. Composition and weight of the medium in the installation.

\begin{tabular}{|l|l|l|l|l|l|}
\hline \multirow{2}{*}{ Unit name } & \multicolumn{3}{|c|}{ Weight of unit layers. kg } & \multirow{2}{\text{Installation}}{$\begin{array}{c}\text { weight. total. } \\
\mathrm{kg}\end{array}$} \\
\cline { 2 - 5 } & Sand & $\begin{array}{c}\text { Cardboard } \\
\text { box }\end{array}$ & $\begin{array}{c}\text { Steel } \\
\text { box }\end{array}$ & Water & \\
\hline Medium - sand & 2.02 & 0.24 & - & - & 2.26 \\
\hline Medium - water & 0.92 & 0.24 & 0.6 & 0.5 & 2.26 \\
\hline
\end{tabular}

Electroacoustic transducer - diffuser brand 4GD-28 with a diameter of $20.2 \mathrm{~cm}$, located perpendicular to the base of the models. The loudspeaker cabinet had an open back wall, with side walls $10 \mathrm{~cm}$ high. The frequency of the mechanical resonance of the loudspeaker, according to the manufacturers, is available at frequencies below $63 \mathrm{~Hz}$. At a frequency of $1000 \mathrm{~Hz}$, the length of the excited wave $\lambda$ was $34.0 \mathrm{~cm}$, and at $10000 \mathrm{~Hz}$, respectively, 3.4 $\mathrm{cm}$.

Table 2. Model specifications.

\begin{tabular}{|c|c|c|c|c|c|c|}
\hline 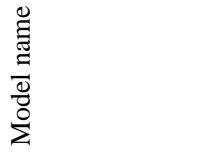 & 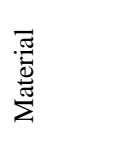 & 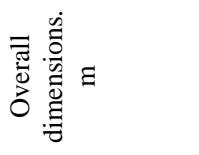 & 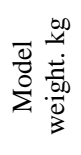 & 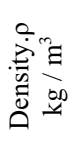 & 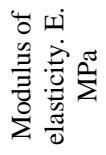 & $\begin{array}{l}n \\
\ddot{0} \\
\dot{0} \\
\dot{0} \\
\dot{0} \\
\dot{0}\end{array}$ \\
\hline Jetty head & Plexiglass & $0.1 * 0.1 * 0.04$ & 0.04 & \multirow{3}{*}{1190} & \multirow{3}{*}{$\begin{array}{c}2800- \\
4900\end{array}$} & \multirow{3}{*}{0.33} \\
\hline 3-storey building & Plexiglass & $0.1 * 0.1 * 0.09$ & 0.098 & & & \\
\hline Cube & Plexiglass & $0.06 * 0.06 * 0.035$ & 0.149 & & & \\
\hline Jetty head & $\mathrm{ABS}$ & $0.1 * 0.1 * 0.04$ & 0.04 & \multirow{3}{*}{$\begin{array}{c}1020- \\
1080\end{array}$} & \multirow{3}{*}{1270} & \multirow{3}{*}{0.37} \\
\hline 3-storey building & ABS & $0.1 * 0.1 * 0.09$ & 0.098 & & & \\
\hline 6-storey building & $\mathrm{ABS}$ & $0.1 * 0.1 * 0.18$ & 0.2 & & & \\
\hline
\end{tabular}


Wave propagation medium - air, sand, distilled water (weight in table 1), models made of plexiglass and ABS plastic (characteristics in table 2). Joints of plexiglas model elements have been adhesive on dichloroethane. Models of three and six storey buildings (Figure 1) in ABS plastic are printed on a 3-D printer, and a model of an ABS jetty head (Figure 2) is glued on dichloroethane.

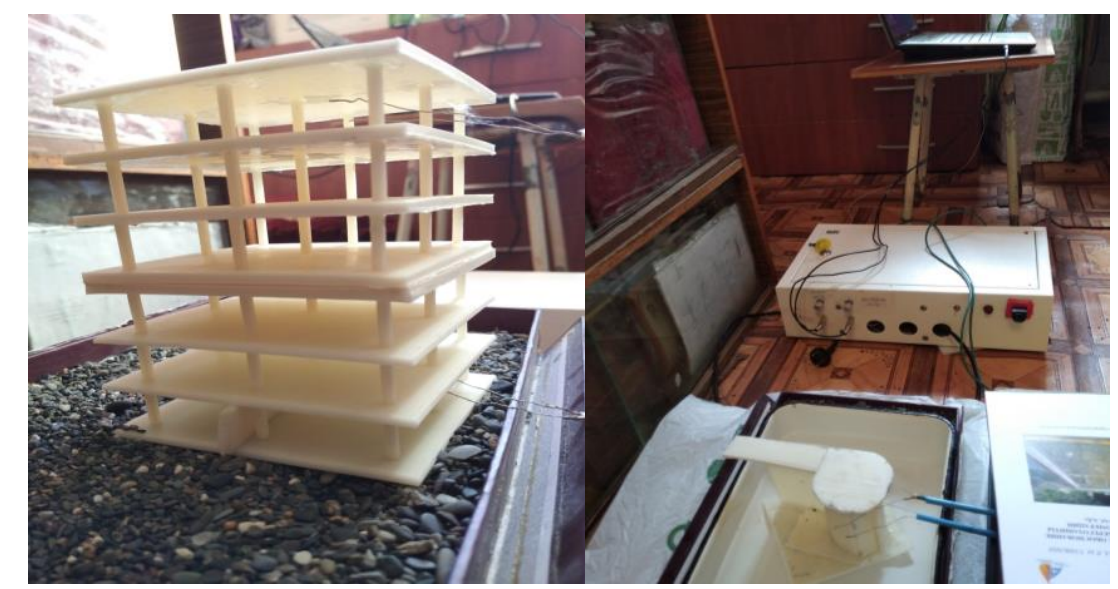

Fig. 1. Six storey building in.

Fig. 2. Jetty head in ABS.

The properties of the air medium and the shape of the wave front are constant in the experiment. The ratio of the diffuser diameter $\mathrm{d}=20.2 \mathrm{~cm}$ to the wavelength $\lambda$ at the generated frequency reflected the dependence of the active and reactive components of the acoustic impedance. The acoustic resistance (impedance) of water is approximately 3600 times the acoustic resistance of air, means that the sound pressure should increase compared to the pressure in the air, which was observed experimentally. According to [7], only a small fraction of the incident wave energy passes into the water from the air, and even less into the sand. The sound energy was completely reflected from the interface of the medium with high acoustic resistance, that is, from sand, and to a lesser extent from water. The values of the wave propagation velocities, acoustic impedances of the medium, according to [7-13] and the materials of the models are presented in Table 3.

Table 3. Wave velocities and acoustic impedance.

\begin{tabular}{|c|c|c|c|c|}
\hline \multirow{2}{*}{ Materials } & \multicolumn{3}{|c|}{ Wave propagation speed, $\mathrm{m} / \mathrm{s}$} & \multirow{2}{*}{$\begin{array}{c}\text { Acoustic impedance, } \\
\mathrm{kg} /(\mathrm{m} 2 * \mathrm{~s})\end{array}$} \\
\cline { 2 - 4 } & Longitudinal & \multicolumn{1}{|c|}{ Shear } & \multicolumn{1}{c|}{ Sound } & \\
\hline Air & - & - & 340 & $0.00033 * 106[9]$ \\
\hline Water & $1400-1500$ & - & 1450 & $1.48 * 106$ \\
\hline Sand & $400-1200$ & $100-$ & $1470-1660$ & $14.2^{*} 106[10]$ \\
\hline Plexiglass & 2680 & 1100 & $119[\mathrm{C}, \mathrm{E}, \mathrm{F}]$ & $3.2213 * 10^{6}[12,13]$ \\
\hline ABS & - & - & $2250[\mathrm{C}, \mathrm{E}, \mathrm{F}]$ & $2.363 * 10^{6}[12,13]$ \\
\hline
\end{tabular}

The scale of the models 3- and 6-storey buildings is M 1: 100, the jetty head - M 1: 300 from the prototypes.

The AC microphone amplifier is two-stage, the gain of a single stage, performed on an operational amplifier, reaches 100,000 times. The connection between the stages is carried 
out: with the help of blocking capacitors, an electrolytic capacitor $47 \mathrm{mF}$ at $25 \mathrm{~V}$ has a low impedance even at low frequencies. Electrodes are coaxial wires connected to the microphone amplifier on one side and loosely attached to the models on the other side.

Computer in oscilloscope mode - potentials are recorded simultaneously from two pairs of electrodes through two channels. The smallest amplitude of the input signal is $200 \mathrm{mV}$, the frequency response of 16 bits of the sound card is $20-20000 \mathrm{~Hz}$ ADC sound card.

Measurement parameters using Spectrolab software - measurement amplitude - from units of microvolts to tens of millivolts. It is not the absolute value of the potential that is measured, but the sum or the difference of potentials at two points of the models during 30$40 \mathrm{~s}$,

Checking and operating the installation - the frequency of sinusoidal oscillations was set on the abscissa axis in the SpectroLab program, and the amplitude of these oscillations on the ordinate axis. Then the frequency at the input was increased without changing the amplitude. Depending on the frequency response of the device, different results were obtained on the recording: in one case the amplitude of the potentials decreased (distorted) at a low frequency, and in the other - at a higher frequency. Leakage currents are minimized as the mains supply is cut off. The equipment is powered by autonomous direct current sources - galvanic batteries and accumulators. The steel case of the microphone amplifier is not only a closed electrostatic shield, but also a magnetic shield. To minimize interference by transmission, the signals are fed to the input of the microphone amplifier using coaxial cables. The output of the microphone amplifier and the line input of the sound card are connected using coaxial cables.

Dynamic actions transmitted through the soil for structures are considered kinematic. The response depends on the characteristics of the base and the features of the design. For modeling, representatives of two different design schemes were selected - frame and frameless in the soil conditions of coastal areas - sea sand and water. Model foundations slab.

Models of frame structures without filling or internal walls have three or six floors, a simple shape in plan. Slabs are considered non-deformable and rigidly connected to the columns. Overlap reactions will be translational movement and rotation in its plane. In mathematical models, as a rule, such design schemes are replaced by an equivalent flat frame with masses and stiffnesses equal to the total masses and total stiffnesses of the corresponding elements in the direction of vibration. The transverse stiffness of the floor slab is taken as the transverse stiffness of the crossbar of such a frame, and the sum of the transverse stiffness of the columns of the tier row is taken as the transverse stiffness of the column. Therefore, in the test circuit, the oscilloscope electrodes were placed near the middle frame of the models. In a three-storey structure, the distance between the electrode at the model support and the top was $4 \mathrm{~cm}$, in a six-storey one $-13 \mathrm{~cm}$.

The model of frameless structures is represented by the jetty head. It has a cylindrical hollow part rigidly connected to the cage. The ABS model has a rigid plate-like base, the plexiglas model - has no base. The walls of the pier, both in the cylindrical part and in the buttress, are rigidly connected with a non-deformable coating. The reactions of the coating will be translational movement in the horizontal plane, the walls work for shear in their plane. In mathematical models, shear vibrations of the walls are taken into account, and the floors move like hard disks. The possibility of vertical, horizontal displacements and turns with non-rigid bases is taken into account. In the test circuit, the oscilloscope electrodes are placed at the walls of the hollow cylindrical part of the jetty head. The distance between the electrode at the model support and the top was $4 \mathrm{~cm}$.

The model in the form of a plexiglass cube simulated a rigid homogeneous monolithic structure and was examined for comparison with the responses of frame and frameless models. Models in the sand medium had free support or pinching, in water - free support. 
They are considered as electromechanical filters (mechanical resonators) capable of converting electrical vibrations into mechanical ones, filtering them and converting them into electrical ones. In the literature [14], mechanical resonators are known as rods, balls, discs, and rectangular thin plates. The quality factor of the resonators considered in [14] makes it possible to obtain bandpass filters with steep slopes and flat tops. Depending on the average frequency and the selected connection scheme of the filter elements, the bandwidth of the mechanical filters is set from tens of hertz to tens of kilohertz.

Such filters are used in radio engineering in the form of horizontally arranged rods and vibration exciters. When the frequencies of the external exciting force coincide with the natural vibration frequency, resonance is observed, at which periodic changes in the length of the rod become maximum.

The natural frequency of rods with both free or fixed ends depends on the length of the rod 1 and on the speed of propagation of mechanical vibrations vpr along its axis:

$$
f n=n \cdot v p r /(2 \cdot l)
$$

where $\mathrm{n}$ - the number of the harmonic at which resonance occurs.

The speed of propagation of mechanical vibrations is expressed through the bulk density of the rod material and the modulus of elasticity E, then:

$$
f n=n \cdot \sqrt{ }(E / \rho) /(2 \cdot l)
$$

The lowest vibration occurs with a frequency equal to vpr / (21), then the length of the rod is equal to half the wavelength $1=\kappa 2$. If both ends of the rod are not fixed, and its length is equal to half the length of the mechanical wave, then in the middle of the rod it will be motionless (node), and at the ends of the antinode. Mechanical filters also use bending vibrations of metal circular plates. The forms of resonant vibrations of free round plates differ in nodal circles (remaining stationary - the center shifts) and nodal diameters (the center remains stationary).

These types of oscillations are excited at different frequencies proportional to the value:

$$
(n+2 h) 2
$$

where:

$\mathrm{n}$ - the number of nodal diameters,

$\mathrm{h}$ - the number of nodal circles.

For $\mathrm{n}=0, \mathrm{~h}=2$, the dependence of the vibration frequency on the ratio of the plate thickness to its radius is plotted. In [14], filters are considered using tensile vibrations of thin metal plates with a large ratio of height $b$ to width $\mathrm{L}$. The dependence of the resonant frequency of the plate on the ratio $\mathrm{mb} / \mathrm{L}$ is presented, where

$\mathrm{m}$ - the number of the excited harmonic to $\sqrt{ }(\mathrm{E} / \mathrm{\rho})$ - the propagation velocity of longitudinal vibrations in a rod made of the plate material. With the selected harmonic number, resonant oscillations can be observed at two frequencies. They are vibrations of the first (lower vibrations) and second (higher) kind. In addition to resonators, mechanical filters include bundles that transmit mechanical vibrations from one resonator to another.

Mechanical resonators with dimensions in terms of wavelengths and consisting of:

- resonators - columns $K / 4$ long, bundles - four slabs $K / 40$ long - in models of 3-storey buildings,

- resonators - stands of plates $K$ long, bundles - overlap plates $K 40$.

- resonator - cube $\kappa 2$ long, there are no bundles.

The dynamic action generated during the tests was set to be sinusoidal, but at the interface between media with different impedances, the waves were divided into transmitted and reflected waves. The vibration amplitudes were attenuated and the shape of the incoming 
signal was distorted. Since the cone structure is a pipe, standing waves arise in rigid obstacles, which leads to numerous resonances. Nodes and antinodes of sound pressure and vibrational velocity are formed in the test setup, it interacts with the sound field over the entire area. The principle of normal impedance is triggered, the resistance of each layer is taken into account. Starting at $5 \mathrm{kHz}$, the wavelength is less than the diameter of the diffuser, the emitted waveform is flat. The wavelengths at frequencies are shown in Table 4.

Table 4. Wavelength in model materials at frequency.

\begin{tabular}{|l|l|l|l|}
\hline Frequency $\mathrm{f}_{0}, \mathrm{kHz}$ & \multicolumn{3}{|l|}{ Wavelength in model materials } \\
\cline { 2 - 4 } & Longitudinal & Shear & \\
\cline { 3 - 4 } & & Plexiglass & ABS \\
\hline 10000 & 0.53 & 0.22 & 0.225 \\
\hline
\end{tabular}

When analyzing the responses of the models by the phase shift between the oscillograms of the bottom and top of the models, only frequencies that are not multiples, are taken into account [15]. When irradiating acoustically "hard" sand and models, the acoustic resistance of which is greater than that of air, see Table 3, the reflection of acoustic signals of multiple frequencies occurs without a phase shift by $\pi$ radians.

\section{Results}

Comparing the data of tables 2 and 4 noticeably, the models are able to filter waves of different directions. Then, the normalized resonant frequency of the models is calculated according to $[14,16]$ :

$$
f=\left(\left(0,5 * E^{*}(1+v) / \rho\right)\right) 0,5 /(2 * t)
$$

provided that $\mathrm{t}$ - is the height of the models and the characteristics from Table 2.

Table 5 compares the calculated and measured multiple frequencies at amplitudes at the maximum phase shift between the oscillograms of the left and right channels. The electrodes of the left channel are placed at a distance of $2 \mathrm{~cm}$ from the model supports, of the right channel at a distance of $6 \mathrm{~cm}$. The models are freely supported on the sand. The frequency response is shown in Figure 3.

Table 5. Models resonant frequencies calculated and measured.

\begin{tabular}{|c|c|c|}
\hline Model name (material) & $\begin{array}{c}\text { Resonant response } \\
\text { frequency f. kHz } \\
\text { (calculated) }\end{array}$ & $\begin{array}{c}\text { Resonant response } \\
\text { frequency f. kHz } \\
\text { (measured) }\end{array}$ \\
\hline Jetty head (plexiglass) & 1.975 & 2.614 .9 .958 \\
\hline Jetty head (ABS) & 1.460 & 1.464 .9 .599 \\
\hline $\begin{array}{c}\text { 3-storey building } \\
\text { (plexiglass) }\end{array}$ & 2.194 & 1.851 \\
\hline 3-storey building (ABS) & 1.622 & 2.539 \\
\hline
\end{tabular}

From the analysis of Figure 3, it is clear that the measuring system is sensitive to the shape of the models, since the amplitudes $(\mathrm{mV})$ along the left and right channels for a 3 storey building differ significantly from the amplitudes for the head of a breakwater. In models of a 3-storey building, attenuation of reactions along the height of the model is observed, and the oscillations of the bottom and top of the breakwater head have practically the same values. This difference in responses is related to the shape of the resonator models. In a 3-storey building, there are four bundles, and one in the head of the breakwater. Figure 1 shows an insignificant difference in the reactions of the models depending on the material 
- plexiglass or plastic. The amplitude values of the bottom and top of the breakwater head from ABS are the same, in contrast to the model made of plexiglass. This is due to the high rigidity of the model in the support, since it has a base plate, but not in the plexiglass model.

We consider the resonance frequencies of the building to be $1.9-2.1 \mathrm{kHz}$, and the jetty head is at frequencies of $1.5-1.9 \mathrm{kHz}$ and $9.5 \mathrm{kHz}$.
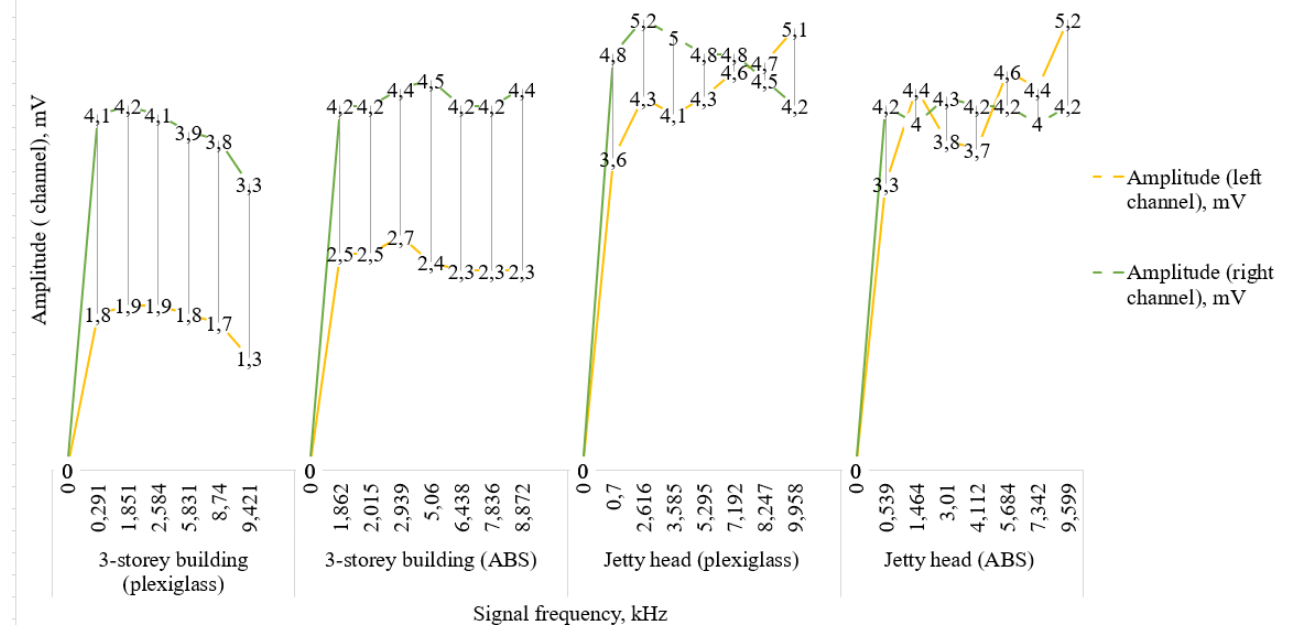

Fig. 3. Frequency response at multiple frequencies.

The estimation of the support conditions by the values of the obtained amplitudes can be produced by the methods of mechanics [5] by the coefficient of dynamic compliance. The calculations are presented in Table 6.

Table 6. Coefficient of dynamic compliance.

\begin{tabular}{|c|c|c|c|}
\hline $\begin{array}{c}\text { Low to high } \\
\text { amplitude jetty head } \\
\text { ratio }\end{array}$ & \multicolumn{2}{|c|}{$\begin{array}{c}\text { K. the coefficient characterizing the dynamic compliance of the model from the } \\
\text { environment and the method of support }\end{array}$} & pinching in the sand \\
\cline { 2 - 4 } & $\begin{array}{c}\text { free support on } \\
\text { the sand }\end{array}$ & free support in water & $17.3 / 2.8=6.17$ \\
\hline $\begin{array}{c}\mathrm{A}_{\mathrm{l}} / \mathrm{A}_{\mathrm{h}}(\max )- \\
\text { plexiglass }\end{array}$ & $2.5 / 2.5=1$ & $6.6 / 2.2=3.03$ & $7.3 / 2.8=2.6$ \\
\hline $\begin{array}{c}\mathrm{A}_{\mathrm{l}} / \mathrm{A}_{\mathrm{h}}(\mathrm{min})- \\
\text { plexiglass }\end{array}$ & $1 / 0.5=2$ & $4.3 / 1.3=3.33$ & $15.3 / 2.8=5.46$ \\
\hline $\begin{array}{c}\mathrm{A}_{\mathrm{l}} / \mathrm{A}_{\mathrm{h}}(\mathrm{mid})- \\
\text { plexiglass }\end{array}$ & $1.7 / 1.5=1.1$ & $15.0 / 2.6=5.7$ & not satisfactory \\
\hline Output & satisfactory & satisfactory & $22.2 / 2.5=8.88$ \\
\hline $\mathrm{A}_{\mathrm{l}} / \mathrm{A}_{\mathrm{h}}(\mathrm{max})-\mathrm{ABS}$ & $2.9 / 1.1=2.6$ & $17.9 / 2.7=6.6$ & $9.2 / 2.5=3.68$ \\
\hline $\mathrm{A}_{\mathrm{l}} / \mathrm{A}_{\mathrm{h}}(\mathrm{min})-\mathrm{ABS}$ & $1.1 / 0.5=2.2$ & $12.9 / 2.5=5.6$ & $17.2 / 2.5=6.88$ \\
\hline $\mathrm{A}_{\mathrm{l}} / \mathrm{A}_{\mathrm{h}}(\mathrm{mid})-\mathrm{ABS}$ & $2 / 1=2$ & $14.0 / 2.5=5.6$ & not satisfactory \\
\hline Output & satisfactory & satisfactory & \\
\hline
\end{tabular}

The sensitivity of measurements to the medium of propagation of sound waves and the method of support is shown in Figure 4: 


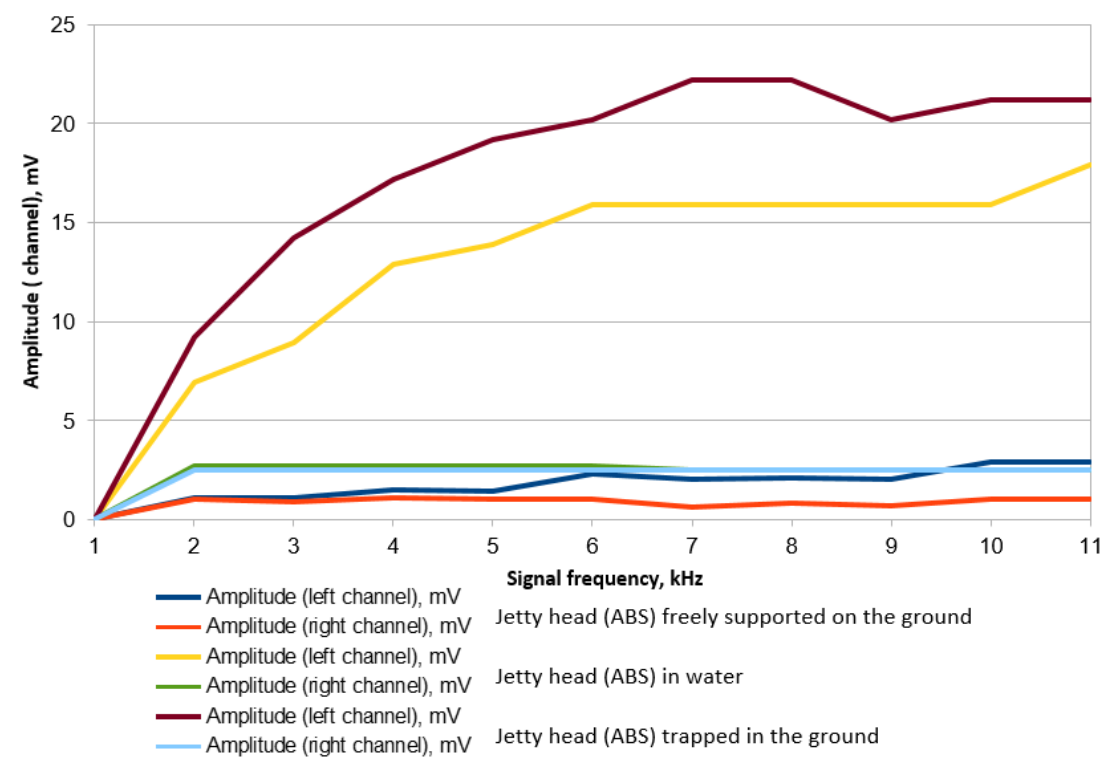

a)

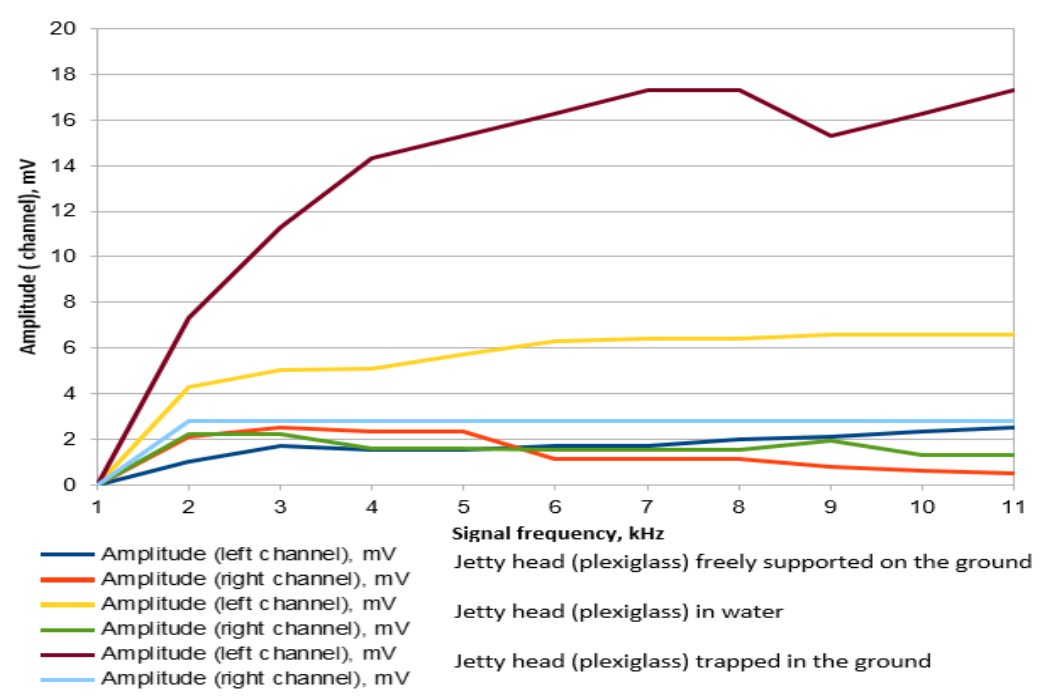

b)

Fig. 4. Frequency response of models in an environment of free bearing on sand, in water, when pinched in sand: a) - AFC of a jetty head made of ABS plastic, b) - AFC of a jetty head made of plexiglass.

The coefficient of dynamic compliance can also serve as a criterion for the quality of connections and nodes. Comparison and connections of options for the quality of support using this coefficient can be used both in design and for assessing existing structures. The analysis consists of the following steps:

- the work of several models is compared, having different support conditions, located in different environments, with different quality of element connections.

- the coefficient $\mathrm{K}=\mathrm{Al} / \mathrm{Ah}$ is determined. The limiting value is $\mathrm{K}<1$, while the average value is

$\mathrm{K}=1$ to 10 .

- the model has an unsatisfactory condition (according to the conditions of the state of the environment or the supporting structure) if the coefficient $\mathrm{K}$ has a decrease of more than $50 \%$ 
in relation to the average value for the same type of models in the same conditions of support and the environment.

The bottom responses of the models demonstrate sensitivity to the wave propagation medium and the way of bearing. The oscillation amplitudes are the greater, the greater the constraint of displacement at the base. With free support on sand, the horizontal movement and rotation of the models are not limited by anything, the acoustic impedance of the medium is air (Table 3). In water with an acoustic impedance higher than that of air, the limitation is immersion in water by $1 \mathrm{~cm}$, and in case of pinching, it is a surcharge with sand. Figure 5 shows the frequency response of the bottom response of the models trapped in the sand. The maximum amplitudes of the response of the models and the diffuser are presented in $\mathrm{dB}$, at frequencies, Hz.

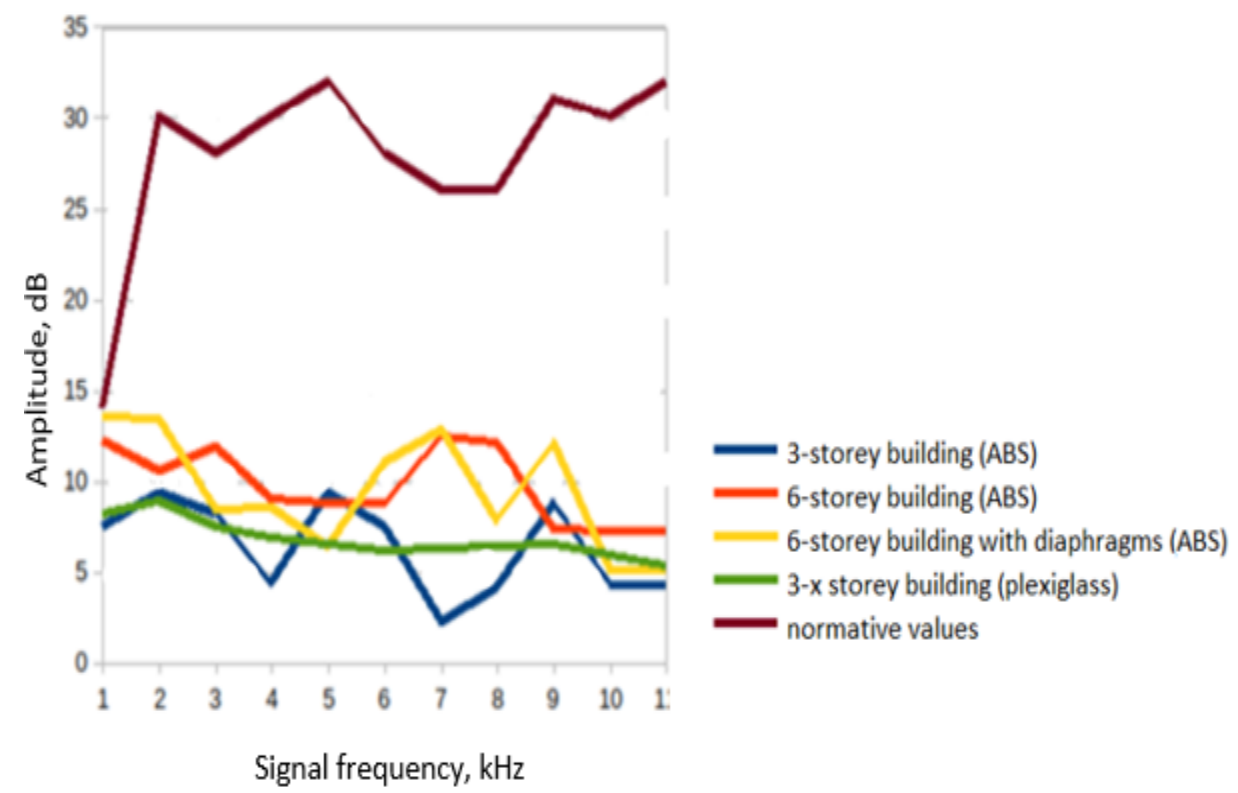

Fig. 5. Frequency response of the bottom response of the models trapped in the sand.

Resonant for models freely supported on sand will be for:

- 3-storey building - frequencies 2.1, $9 \mathrm{~Hz}$ (plexiglass), 2.1, 4.7, $9 \mathrm{~Hz}$ (ABS),

- 6-storey building without diaphragms on the ground floor - frequencies $3,7,7.6 \mathrm{~Hz}$ (ABS).

- 6-storey building with diaphragms on the ground floor - frequencies 1.9, 7, $9 \mathrm{~Hz}$ (ABS).

As follows from the analysis of the graph, the test system turns out to be sensitive to the number of storeys of the models and to the rigidity. The amplitudes of a 6 -storey building are 1.5 times higher than the amplitudes of a 3-storey building; the installation of diaphragms leads to the appearance of a larger number of resonant frequencies.

\section{Conclusions}

1. Received responses of models of structures during testing, sensitive to their shape, soil conditions and method of support.

2. The criteria for assessing the quality by the value of the coefficient of dynamic compliance, obtained from the values of the amplitude of oscillations at multiple frequencies, have been determined. 
3. The use of models as mechanical resonators (filters of certain frequencies) allows the design to exclude resonance phenomena in prototypes. This is done by changing the model so that instead of several resonant frequencies, it filters only one.

\section{References}

1. Wolfson I I 2017 A short course in the theory of mechanical vibrations (Moscow: VNTR Publishing House) p 241

2. O'Toole Ronald P 1991 Characterization and analysis of the Bulk-Acoustic-Wave Thin Film Resonator in sub-nanogram mass detection (Retrospective Theses and Dissertations) https://lib.dr.iastate.edu/rtd/16963

3. Vasina M, Monkova K, Pavol Monka, Kozak P, Tkac J 2020 Study of the Sound Absorption Properties of 3D-Printed Open-Porous ABS Material Structures (Basel: Polymers) doi: 10.3390/polym12051062

4. Rodrigues G, Silva M, Oliveira L 2019 Lat. Am. j. solids struct. 16(7) e204

5. 1987 Theory of Mechanisms and Machines (Moscow: Higher School) p 496

6. Makarov K, Yurchenko E, Yurchenko E 2018 MATEC Web of Conferences IPICSE2018 https://doi.org/10.1051/matecconf/201825104001

7. Kolesnikov Yu, Mednykh D 2004 Physical Mesomechanics 769

8. Burkov S, Sorokin B 2012 Journal of Siberian Federal University. Mathematics \& Physics 5(2) 164

9. Surzhikov A 2018 Determination of the speed of sound propagation in metallic and non-metallic materials https://portal.tpu.ru/SHARED/l/LEXK/lessons/UT/Metodich/Speed.PDF

10. Alabi A, Enikanselu P 2019 Journal of Petroleum Exploration and Production Technology 92487

11. Tarrazó-Serrano D, Pérez-López S, Candelas P, Uris A and Rubio C 2019 Acoustic Focusing Enhancement In Fresnel Zone Plate Lenses doi: 10.1038/s41598-01943495-x

12. Veneziani G, Lima E, Potiens C, Campos L 2016 J. Phys.: Conf. Ser. DOI: $10.1088 / 1742-6596 / 733 / 1 / 012088$ 6596/733/1/012088

13. Minin I, Minin O 2018 MATEC Web of Conferences https://doi.org/10.1051/matecconf/201815501029IME\&T

14. Golubtsov M G 1957 Electromechanical RF Filters (Moscow-Leningrad: SEI Publishing House)

15. Kuphaldt $\mathrm{T}$ 2000-2015 Lessons In Electric Circuits openbookproject.net/electricCircuits

16. Mikushin A V, Sedinin V I 2017 Schematic and system engineering of electronic means (Novosibirsk: Publishing house SibSUTI) 